\title{
Análisis de la producción de fibra de vicuñas en semicautiverio y silvestría en Apurímac, Perú
}

\author{
Analysis of vicuña fibre production in semi-captivity and wild in \\ Apurímac, Peru
}

\author{
Miguel A. Valenzuela-Pinares ${ }^{1}$, Víctor Alberto Ramos De la Riva ${ }^{1,3}$, \\ Ludwing A. Cárdenas-Villanueva ${ }^{1}$, Sergio Pezo-Carreón ${ }^{2}$
}

\section{Resumen}

\begin{abstract}
El estudio tuvo como objetivo analizar la producción de fibra de vicuña (Vicugna mensalis mensalis) en semicautiverio y silvestría entre 2008 y 2016 en la región Apurímac, Perú, así como determinar la población de vicuñas capturadas y esquiladas y el efecto del sexo, edad y sistema de manejo en la producción de fibra. La producción de fibra de vicuña en los nueve años del estudio fue de $2623 \mathrm{~kg}$, promedio anual de $295.7 \mathrm{~kg}$, rendimiento de $152.76 \mathrm{~g}$ /vicuña esquilada y un rango de variación de 54-426 g. La producción de fibra tuvo una tasa de crecimiento anual de $9.45 \%$ y una tasa de captura de $10.34 \%$. Al análisis factorial se encontró diferencia significativa $(\mathrm{p}<0.05)$ para las interacciones años, edad, sexo y efectos simples.
\end{abstract}

Palabras clave: chacu; rendimiento; captura; esquila; vellón

\section{AbSTRaCT}

The aim of this study was to analyse the production of vicuña (Vicugna mensalis mensalis) fibre in semi-captivity and wild between 2008 and 2016 in the Apurímac region of Peru, as well as to determine the population of vicunas captured and sheared and the effect of sex, age and management system in the fibre production. The production of vicuña fibre in the nine years of the study was $2623 \mathrm{~kg}$, annual average of $295.7 \mathrm{~kg}$, yield of $152.76 \mathrm{~g} /$ vicuña and a range of variation of 54-426 g. Fibre production had an annual growth rate of $9.45 \%$ and a capture rate of $10.34 \%$. Factorial analysis showed a significant difference $(\mathrm{p}<0.05)$ for the interactions of years, age, sex and simple effects.

Key words: chacu; performance; capture; shearing; fleece

\footnotetext{
${ }^{1}$ Universidad Nacional Micaela Bastidas de Apurimac, Perú

2 IVITA Maranganí, Universidad Nacional Mayor de San Marcos, Cusco, Perú

${ }^{3}$ E-mail: ramosdelarivavictor@gmail.com
}

Recibido: 21 de enero de 2019

Aceptado para publicación: 2 de noviembre de 2019 


\section{INTRODUCCIÓN}

La vicuña es el camélido sudamericano con mejor adaptación al ecosistema de altiplano andino (Bonacic, 2000). Es un animal silvestre de fácil captura, manipulación y esquila, potencialmente disponible para un crecimiento poblacional sostenido y constituye un recurso natural renovable de gran potencial para el Perú, quién posee el $80 \%$ de la población mundial de esta especie. Es además una alternativa socioeconómica para el poblador altoandino (Lichtenstein et al., 2006). El promedio de producción es de $250 \mathrm{~g}$ de fibra fina por vicuña cada dos años (Brack, 1980).

El color del vellón es canela y se distribuye en forma única y uniforme por el cuerpo del animal, característica que le permite una perfecta mimetización en el paisaje de las punas altoandinas (Calle, 1982). Lo más relevante de la producción de fibra de vicuña es la calidad, la cual es una de las mejores del mundo, de allí la necesidad de buscar y asegurar la producción de fibra de vicuña sin alterar sus poblaciones y capacidad reproductiva (Zúñiga y Bujaico, 2014).

La situación actual de las poblaciones de vicuña, luego de intensos esfuerzos de protección, presenta una serie de nuevos desafios (Gimpel y Bonacic, 2006). Probablemente el más importante sea cómo lograr un beneficio para compensar el esfuerzo de conservarlas, así como el uso y la producción adecuada de su fibra (Rabinovich et al., 1991). Hasta el día de hoy, la producción de fibra se lleva a cabo usualmente mediante la captura y esquila anual de las vicuñas, actividad que se realiza entre mayo y noviembre, con el objetivo de tener una mejor vigilancia, esquilándose solo aquellos animales con fibras no menores de $2 \mathrm{~cm}$ de largo (Lichtenstein et al., 2002).
El aumento de la producción de fibra y la conservación del recurso genético animal, que a la vez permita mejorar la calidad de vida de los pequeños productores, debe ser parte de una estrategia global de inversión sostenida en investigación y de desarrollo apropiado (Quispe et al., 2009). En este contexto, para esta investigación se analizó la producción de fibra de vicuña, determinando la población de vicuñas capturadas, esquiladas y la interacción entre los factores sistema de manejo, edad y sexo en la producción de fibra entre 2008 y 2016.

\section{Materiales y Métodos}

La población de estudio estuvo constituida por vicuñas (Vicugna mensalis mensalis) que habitan en las seis provincias de la región de Apurímac, Perú (Abancay, Andahuaylas, Cotabambas, Grau, Antabamba, Aymaraes) y sus respectivos comités de manejo de vicuña y asociaciones vicuñeras. La zona se encuentra ubicada a una altitud que varía entre 3800 y $4800 \mathrm{msnm}$, con temperaturas que descienden hasta $-10^{\circ} \mathrm{C}$. La temporada de esquila de vicuñas comienza en el mes de mayo y termina en el mes de noviembre según la programación de captura y esquila de la Dirección de Camélidos de la Dirección Regional Agraria Apurímac, en coordinación con los comités de manejo de vicuña.

El manejo de las vicuñas en semicautiverio se realizó en espacios limitados por barreras físicas artificiales (mallas ganaderas) bajo un plan de manejo aprobado. En el caso de animales en silvestría, se consideró a los animales que viven libremente en el territorio de la región Apurímac (MINAGRI, SERFOR, 2015). Las vicuñas se caracterizan de acuerdo con la edad en vicuñas crías: ejemplares de ambos sexos, antes de su madurez sexual, o sea antes de 
los 12 meses; vicuñas juveniles: hembras entre los 12 meses de edad y el primer parto; machos entre los 12 meses de edad y su salida de la tropilla como juveniles; y vicuñas adultas: hembras después del primer parto y los machos después de su salida de la tropilla juvenil (Cajahuamán, 2018).

Se utilizó el muestreo por conveniencia, una técnica de muestreo no probabilístico donde las muestras fueron seleccionadas con base a su accesibilidad. Debido a esto, se tomó en cuenta los registros de captura y esquila de vicuñas de los años 2008 al 2016, que en total comprendieron 54486 vicuñas con 17174 datos productivos sobre peso de vellón sucio de vicuña.. Para obtener la tasa de captura se tomó en cuenta el valor inicial (Vo) año 2008; valor final $\left(V_{f}\right)$ año 2016 y los 9 años de análisis (y) a través de la siguiente ecuación:

$$
\text { Tasa Captura }=\left[\left(\frac{V_{f}}{V_{0}}\right)^{1 / y}-1\right] \times 100
$$

Para el análisis de la producción de fibra (peso de vellón) se utilizó estadística descriptiva (análisis de dispersión y tendencia central), histogramas, tasa de captura con los registros y consolidados de captura y esquila de vicuñas, tanto en el sistema de manejo de silvestría como de semicautiverio, determinándose la población de vicuñas capturadas y esquiladas con los datos contenidos en los registros de captura y esquila reportados por la Dirección de Camélidos de la Dirección Regional Agraria Apurímac. Se analizaron los efectos simples de los sistemas de manejo, edad y sexo utilizando un nivel de confianza del 95\%.

\section{Resultados y Discusión}

Se capturaron 36737 vicuñas en el periodo de estudio (Figura 1), siendo $13358 \mathrm{y}$ 12053 en las provincias de Aymaraes y Andahuaylas, respectivamente, seguido por Cotabambas con 6407, Grau con 4093,
Antabamba con 491 y Abancay con 335. El número de capturas se fue incrementando durante este periodo (desde 16 en 2008 hasta 27 capturas en 2015 y 2016), obteniéndose una tasa de captura de $11.6 \%$, dada la importancia que las comunidades campesinas le están dando a la vicuña y a la comercialización de la fibra como fuente de ingreso económico adicional para la comunidad.

El número de vicuñas esquiladas fue de 17174 vicuñas (6576 en Aymaraes, 6188 en Andahuaylas, 2063 en Cotabambas, 1836 en Grau, 313 en Antabamba, 198 en Abancay) (Figura 2). No todas las vicuñas capturadas son esquiladas, pues solo se esquilan las vicuñas que tienen la longitud de mecha y la edad adecuada. Las vicuñas que no cumplen estas características son liberadas para ser esquiladas en los siguientes programas de captura y esquila.

Pese a que existe un incremento en el número de vicuñas como de esquiladas, existen áreas con poblaciones de vicuñas sin declarar, las cuales incrementarían el aprovechamiento y manejo de la vicuña en la región, tal como lo manifiesta MINAGRIDGFFS (2014) en el censo nacional de 2012, donde se reportan 9982 vicuñas entre juveniles y adultos distribuidas en las seis provincias de la región de Apurímac.

La producción de fibra sucia de vicuña del 2008 al 2016 (Figura 3) fue $191.09 \mathrm{~kg}$ al inicio y $430.89 \mathrm{~kg}$ en 2016 , siendo el promedio productivo de $327.93 \mathrm{~kg}$ de fibra sucia por año. La producción de fibra durante el periodo del estudio se duplicó, mostrando una tendencia ascendente con una tasa de crecimiento anual de producción de $9.45 \%$. El rendimiento de fibra (Cuadro 1) del peso de vellón sucio (PVS) fue $152.76 \pm 38.85 \mathrm{~g}$ por vicuña.

El número de vicuñas capturadas según el sistema de manejo fue de 31574 en semicautiverio y de 5163 en silvestría durante el periodo de estudio, de las cuales se es- 


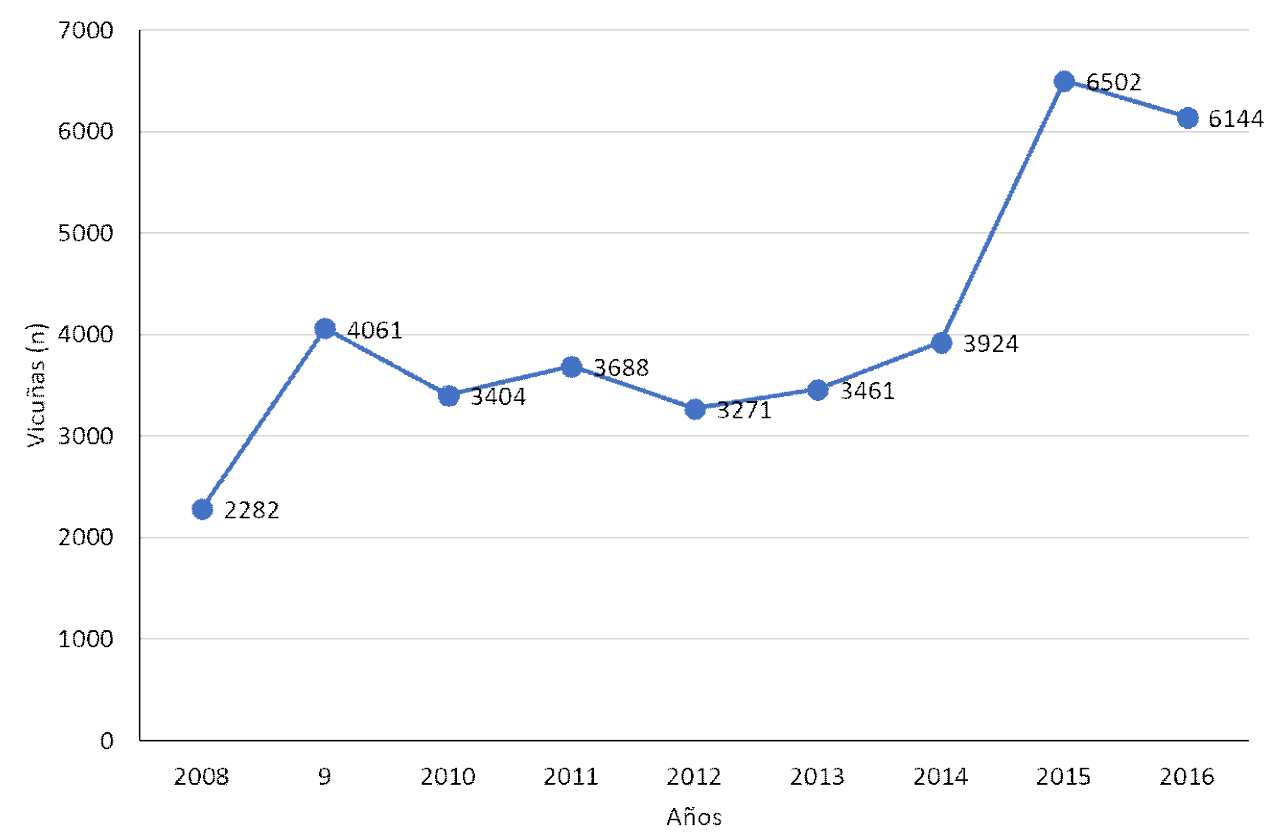

Figura 1. Vicuñas capturadas para la esquila entre los años 2008 y 2016 en la Región Apurímac, Perú

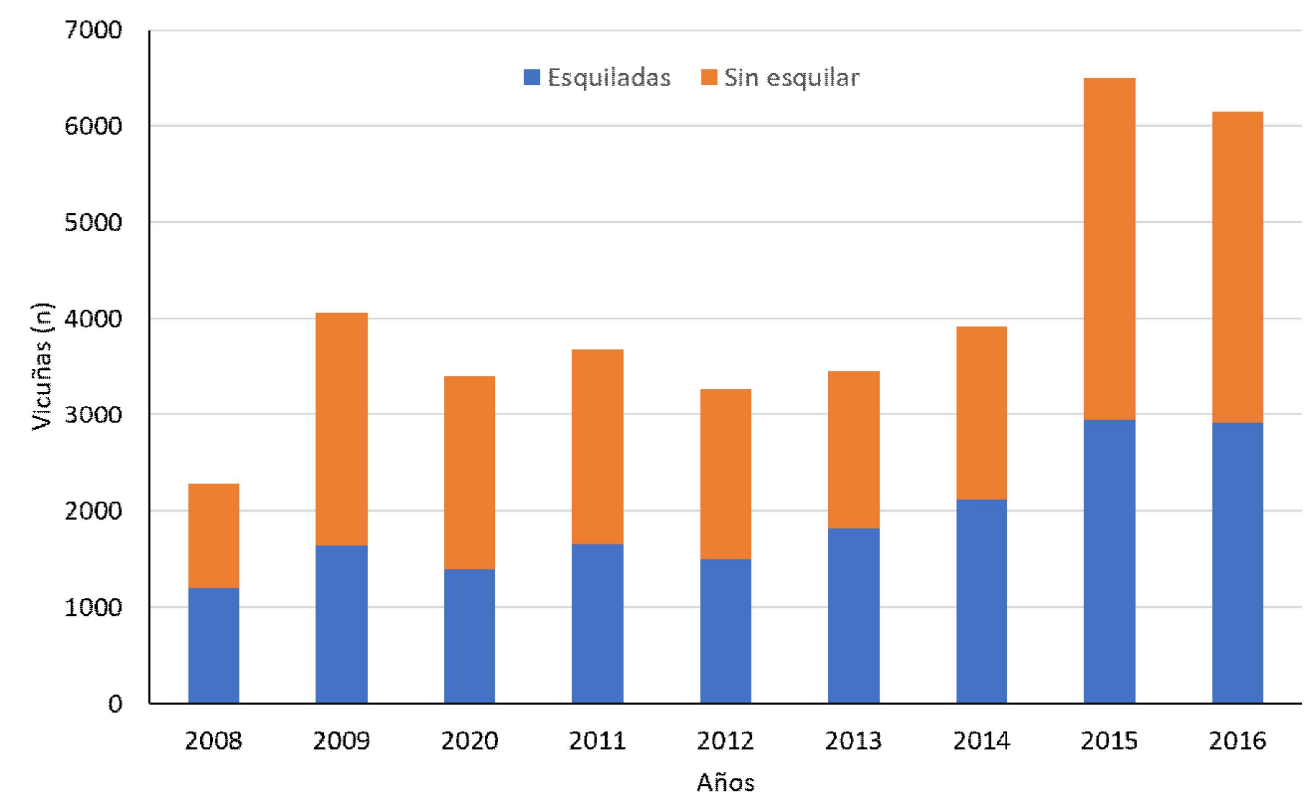

Figura 2. Vicuñas esquiladas y sin esquilar entre los años 2008 y 2016 en la Región Apurímac, Perú 


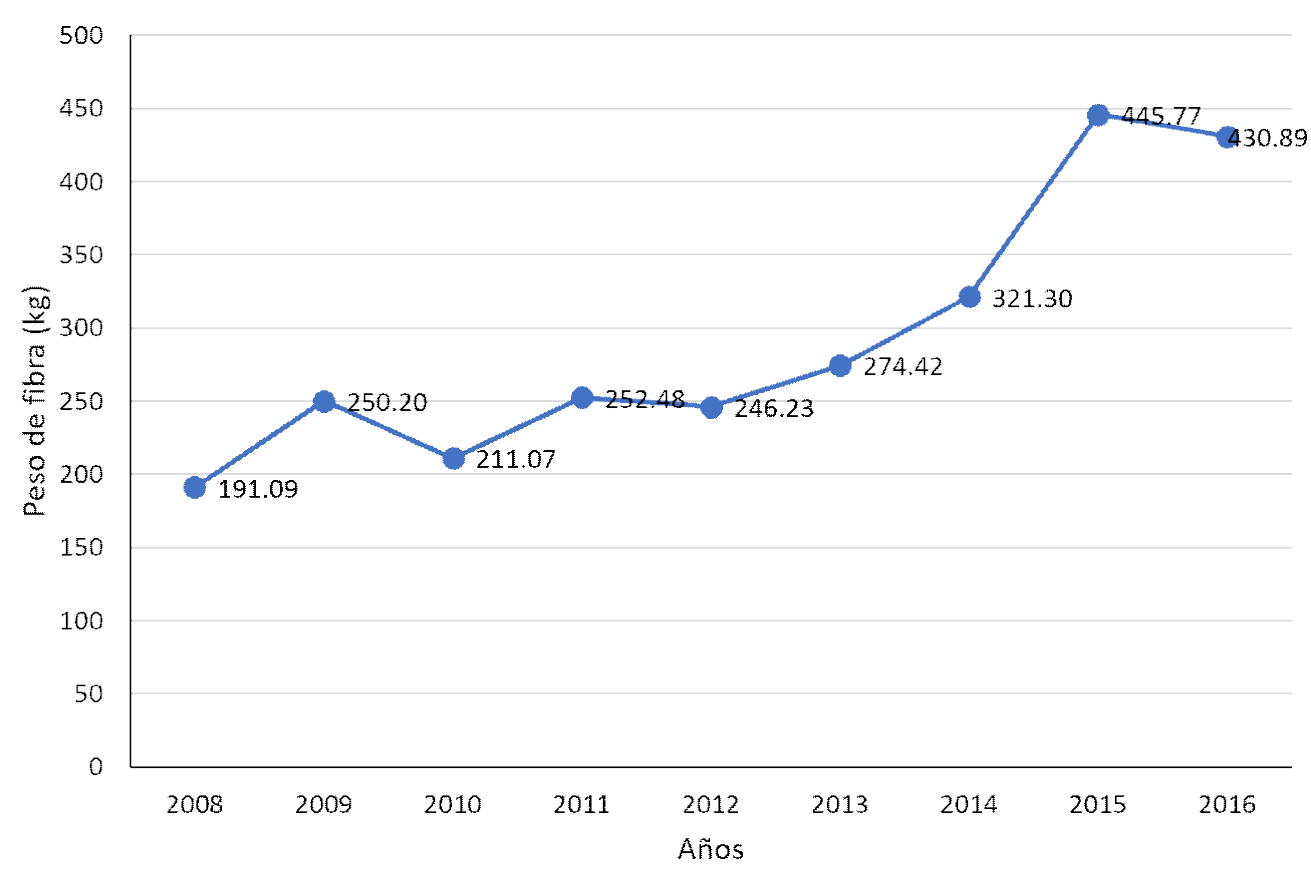

Figura 3. Producción de fibra de vicuñas (kg) entre los años 2008 y 2016 en la Región Apurímac, Perú

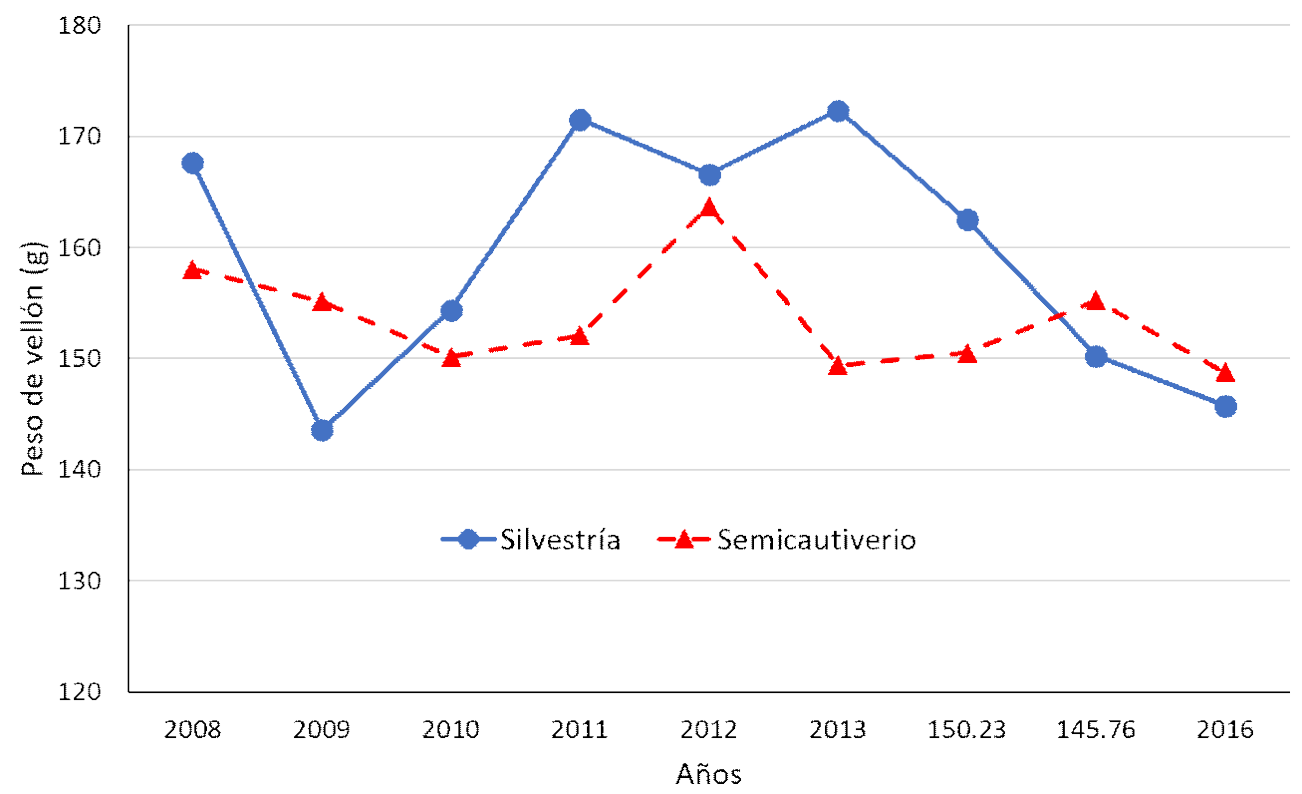

Figura 4. Peso de vellón por sistema de manejo entre los años 2008 y 2016 en la Región Apurímac, Perú 

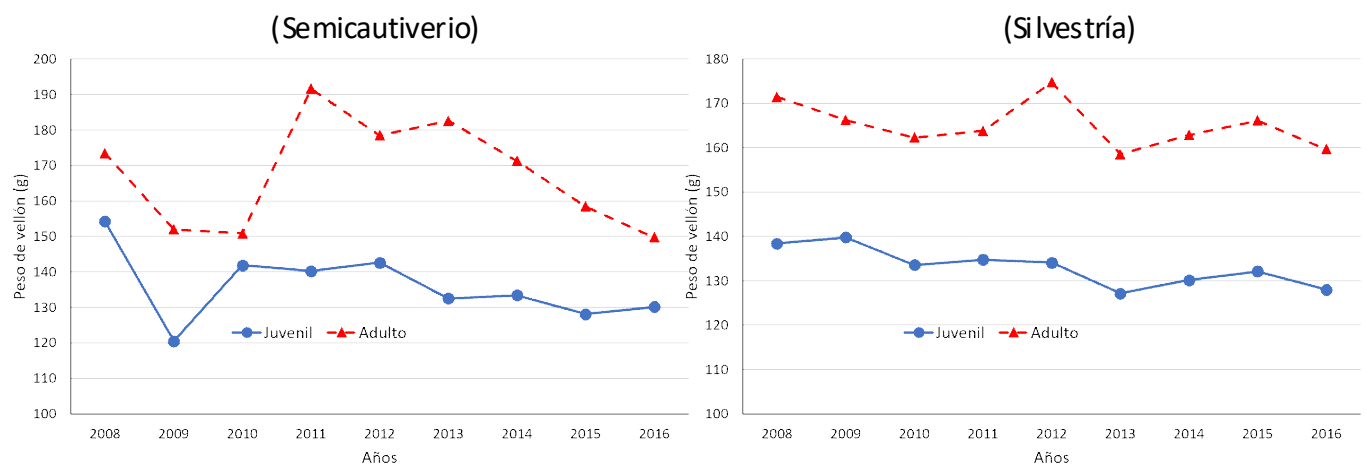

Figura 5. Promedio de pesos de vellón sucio según grupo etario por sistema de manejo entre los años 2008 y 2016 en la Región Apurímac, Perú

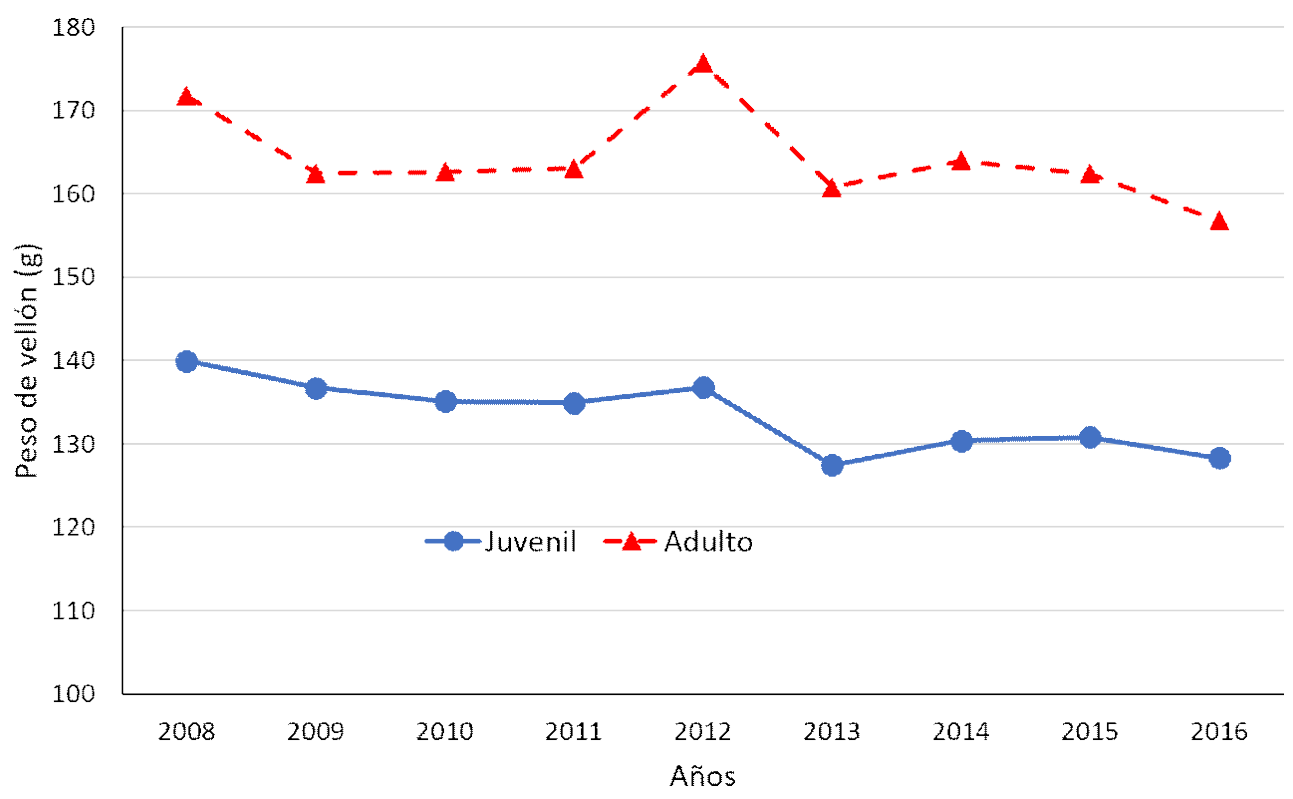

Figura 6. Peso de vellón sucio según grupo etario entre los años 2008 y 2016 en la Región Apurímac, Perú, 


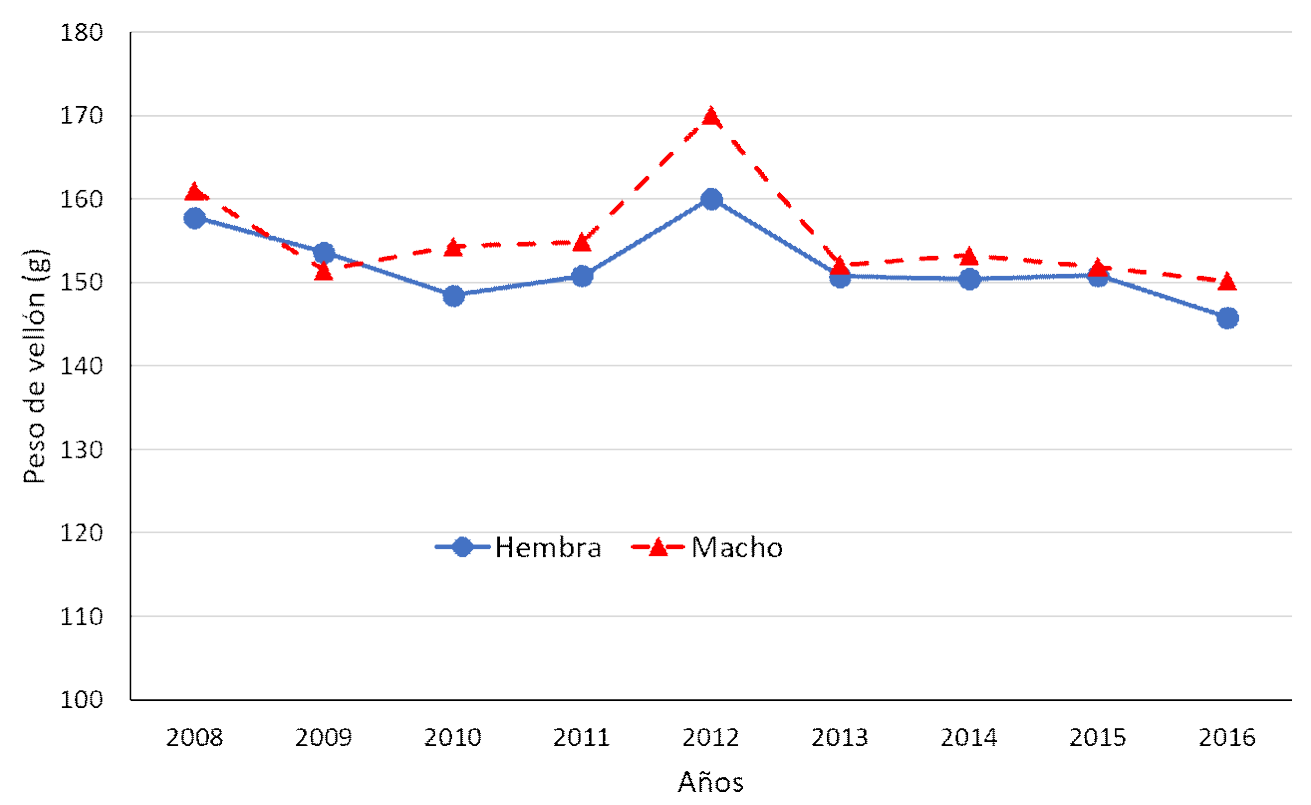

Figura 7. Peso de vellón sucio (PVS) según sexo entre los años 2008 y 2016 en la Región Apurímac, Perú

quilaron 13482 en semicautiverio y 3692 en silvestría (Cuadro 2). Estos valores indican que se capturó el $85.95 \%$ de los animales criados en semicautiverio y el $14.05 \%$ en silvestría.

La producción de fibra expresada en peso de vellón sucio (PVS) no presentó variaciones entre sistemas de manejo al promediar los valores de todo el periodo de estudio (Cuadro 3). Sin embargo, en la interacción entre los sistemas de manejo y los años se observó diferencia significativa $(\mathrm{p}<0.05)$ para los años 2008, 2009, 2011, 2013 y 2014 (Figura 4). Estas diferencias se deberían a que las vicuñas en silvestría tienen menos probabilidades de volver a ser capturadas después de la esquila en comparación a las vicuñas en semicautiverio, lo que les da más tiempo para que se desarrolle el vellón.

En la interacción múltiple sistema de manejo, año y edad se encontró diferencia significativa $(\mathrm{p}<0.05)$ para el efecto edad sobre el sistema de manejo para cada año de estudio. Los pesos de vellón sucio por edad (adulto y juvenil) fueron diferentes en ambos sistemas de manejo y en todo el periodo de estudio (Figura 5).

En cuanto a la población de vicuñas capturadas según edad, se encontró un mayor número de vicuñas adultas, seguido de vicuñas en edad juvenil y crías (Cuadro 4). Cabe señalar que solo para efectos de captura se utilizó el número de crías, pues no se realiza la esquilas en animales de este grupo etario (MINAGRI - DGFFS, 2015). El número de vicuñas adultas esquiladas fue de 11241 , lo cual representa el $65.4 \%$ de la población esquilada (Cuadro 5). Las vicuñas de edad juvenil esquiladas fueron de 5933 (34.6\%) (Cuadro 5).

La producción de PVS (Cuadro 6) fue significativamente mayor en animales adultos que en juveniles $(\mathrm{p}<0.05)$, donde además existió efecto del año sobre el grupo etario $(\mathrm{p}<0.05)$ (Figura 6). 
Cuadro 1. Rendimientos anuales de peso de vellón sucio (PVS) de vicuñas (kg) entre los años 2008 y 2016 en la Región Apurímac, Perú

\begin{tabular}{ccccc}
\hline Año & Vicuñas $(\mathrm{n})$ & Promedio $(\mathrm{g})$ & Rango $(\mathrm{g})$ & CV $(\%)$ \\
\hline 2008 & 1200 & $159.24 \pm 41.38$ & $74-381$ & 26 \\
2009 & 1640 & $152.56 \pm 43.07$ & $55-355$ & 28 \\
2010 & 1396 & $151.19 \pm 39.08$ & $66-330$ & 26 \\
2011 & 1655 & $152.63 \pm 40.79$ & $64-310$ & 27 \\
2012 & 1497 & $164.49 \pm 43.75$ & $76-382$ & 27 \\
2013 & 1813 & $151.36 \pm 40.32$ & $54-346$ & 27 \\
2014 & 2117 & $151.77 \pm 42.92$ & $64-374$ & 28 \\
2015 & 2945 & $151.36 \pm 41.40$ & $64-426$ & 27 \\
2016 & 2911 & $148.02 \pm 38.40$ & $57-356$ & 26 \\
\hline Promedio & & $152.76 \pm 38.85$ & & 24.9 \\
\hline
\end{tabular}

CV $(\%)=$ coeficiente de variación

Cuadro 2. Población de vicuñas capturas y esquiladas según el sistema de manejo, entre los años 2008 y 2016 en la Región Apurímac, Perú

\begin{tabular}{ccccc}
\hline \multirow{2}{*}{ Años } & \multicolumn{2}{c}{ Silvestría } & \multicolumn{2}{c}{ Semicautiverio } \\
\cline { 2 - 5 } & Capturados & Esquilados & Capturados & Esquilados \\
\hline 2008 & 176 & 151 & 2169 & 1049 \\
2009 & 421 & 363 & 3640 & 1277 \\
2010 & 476 & 342 & 2928 & 1054 \\
2011 & 55 & 46 & 3633 & 1609 \\
2012 & 678 & 408 & 2593 & 1089 \\
2013 & 172 & 156 & 3289 & 1657 \\
2014 & 231 & 225 & 3693 & 1892 \\
2015 & 1938 & 1267 & 4564 & 1678 \\
2016 & 1079 & 734 & 5065 & 2177 \\
\hline Total & 5163 & 3692 & 31574 & 13482 \\
\hline
\end{tabular}

Cuadro 3. Peso de vellón sucio de vicuñas según el sistema de manejo

\begin{tabular}{cccc}
\hline $\begin{array}{c}\text { Sistema de } \\
\text { manejo }\end{array}$ & $\begin{array}{c}\text { Vicuñas } \\
(\mathrm{n})\end{array}$ & $\begin{array}{c}\text { Promedio } \\
(\mathrm{g})\end{array}$ & $\begin{array}{c}\mathrm{CV} \\
(\%)\end{array}$ \\
\hline $\begin{array}{c}\text { Silvestría } \\
\begin{array}{c}\text { Semi- } \\
\text { cautiverio }\end{array}\end{array}$ & 3,692 & $153.5 \pm 40.7$ & 26.50 \\
$\mathrm{CV}(\%)=$ coeficiente de variación & $152.6 \pm 41.1$ & 26.93 \\
\hline
\end{tabular}

La población de vicuñas capturadas por sexo fue 20403 machos (45\%) y 25105 hembras $(55 \%)$, proporciones que se mantuvieron durante los ochos años de estudio (Cuadro 7). La población de vicuñas esquiladas fue 8131 machos (47\%) y 9043 hembras $(53 \%)$, proporciones que igualmente se mantuvieron a través de los años (Cuadro 7). 
Cuadro 4. Población de vicuñas capturadas según grupo etario entre los años 2008 y 2016 en la Región Apurímac, Perú

\begin{tabular}{ccccccc}
\hline \multirow{2}{*}{ Años } & \multicolumn{2}{c}{ Adultos } & \multicolumn{2}{c}{ Juveniles } & \multicolumn{2}{c}{ Crías } \\
\cline { 2 - 7 } & $\mathrm{n}$ & $\%$ & $\mathrm{n}$ & $\%$ & $\mathrm{n}$ & $\%$ \\
\hline 2008 & 1791 & 69.0 & 491 & 18.9 & 315 & 12.1 \\
2009 & 3429 & 68.9 & 632 & 12.7 & 916 & 18.4 \\
2010 & 2811 & 67.8 & 592 & 14.3 & 745 & 18.0 \\
2011 & 3075 & 66.5 & 613 & 13.3 & 936 & 20.2 \\
2012 & 2859 & 69.4 & 431 & 10.5 & 831 & 20.2 \\
2013 & 2933 & 68.0 & 513 & 11.9 & 868 & 20.1 \\
2014 & 3142 & 65.0 & 782 & 16.2 & 908 & 18.8 \\
2015 & 5442 & 68.3 & 1029 & 12.9 & 1502 & 18.8 \\
2016 & 5249 & 69.0 & 895 & 11.8 & 1464 & 19.2 \\
\hline
\end{tabular}

Cuadro 5. Población de vicuñas esquiladas según grupo etario entre los años 2008 y 2016 en la Región Apurímac, Perú

\begin{tabular}{ccccc}
\hline \multirow{2}{*}{ Años } & \multicolumn{3}{c}{ Adultos } & \multicolumn{3}{c}{ Juveniles } \\
\cline { 2 - 5 } & $\mathrm{n}$ & $\%$ & $\mathrm{n}$ & $\%$ \\
\hline 2008 & 728 & 60.7 & 472 & 39.3 \\
2009 & 1008 & 61.5 & 632 & 38.5 \\
2010 & 816 & 58.5 & 580 & 41.6 \\
2011 & 1041 & 62.9 & 614 & 37.1 \\
2012 & 1066 & 71.2 & 431 & 28.8 \\
2013 & 1300 & 71.7 & 513 & 28.3 \\
2014 & 1348 & 63.7 & 769 & 36.3 \\
2015 & 1918 & 65.1 & 1027 & 34.9 \\
2016 & 2016 & 69.3 & 895 & 30.8 \\
\hline
\end{tabular}

Cuadro 6. Peso de vellón sucio de vicuñas según el grupo etario

\begin{tabular}{cccc}
\hline $\begin{array}{c}\text { Sistema de } \\
\text { manejo }\end{array}$ & $\begin{array}{c}\text { Vicuñas } \\
(\mathrm{n})\end{array}$ & $\begin{array}{c}\text { Promedio } \\
(\mathrm{g})\end{array}$ & $\begin{array}{c}\mathrm{CV} \\
(\%)\end{array}$ \\
\hline Adulto & 11,241 & $163.4 \pm 41.9^{\mathrm{a}}$ & 25.70 \\
Juvenil & 5,933 & $132.7 \pm 30.6^{\mathrm{b}}$ & 23.03 \\
\hline CV (\%) = coeficiente de variación \\
a,b Diferentes superíndices indican diferencia \\
significativa ( $\mathrm{p}<0.05)$
\end{tabular}

La producción de PVS fue significativamente mayor en machos $(154.72 \pm 42.61$ g) que en hembras $(151.40 \pm 39.66 \mathrm{~g})$ $(\mathrm{p}<0.05$; Cuadro 8). No obstante, el efecto año sobre el factor sexo solo fue significativo $(\mathrm{p}<0.05)$ en los años 2010, 2011,2012, 2016 (Figura 7).

En la interacción sexo (macho y hembra) por edad (adulto y juvenil) se encontró diferencia significativa entre sexo con edad 
Cuadro 7. Población de vicuñas capturadas y esquiladas según sexo entre los años 2008 y 2016 en la Región Apurímac, Perú

\begin{tabular}{ccccccccc}
\hline & \multicolumn{3}{c}{ Vicunas capturadas } & \multicolumn{4}{c}{ Vicunas esquiladas } \\
Años & \multicolumn{3}{c}{ Machos } & \multicolumn{2}{c}{ Hembras } & \multicolumn{2}{c}{ Machos } & \multicolumn{2}{c}{ Hembras } \\
\cline { 2 - 9 } & $\mathrm{n}$ & $\%$ & $\mathrm{n}$ & $\%$ & $\mathrm{n}$ & $\%$ & $\mathrm{n}$ & $\%$ \\
\hline 2008 & 1214 & 41.7 & 1697 & 58.3 & 535 & 44.6 & 665 & 55.4 \\
2009 & 2400 & 48.2 & 2577 & 51.8 & 796 & 48.5 & 844 & 51.5 \\
2010 & 1799 & 43.4 & 2349 & 56.6 & 639 & 45.8 & 757 & 54.2 \\
2011 & 1989 & 43.0 & 2635 & 57.0 & 749 & 45.3 & 906 & 54.7 \\
2012 & 1832 & 44.5 & 2289 & 55.5 & 663 & 44.3 & 834 & 55.7 \\
2013 & 1931 & 44.8 & 2383 & 55.2 & 838 & 46.2 & 975 & 53.8 \\
2014 & 2141 & 44.3 & 2691 & 55.7 & 1015 & 48.0 & 1102 & 52.1 \\
2015 & 3666 & 46.0 & 4307 & 54.0 & 1449 & 49.2 & 1496 & 50.8 \\
2016 & 3431 & 45.1 & 895 & 54.9 & 1447 & 49.7 & 1464 & 50.3 \\
\hline
\end{tabular}

Cuadro 8. Peso de vellón sucio de vicuñas según sexo

\begin{tabular}{cccc}
\hline Sexo & $\begin{array}{c}\text { Vicuñas } \\
(\mathrm{n})\end{array}$ & $\begin{array}{c}\text { Promedio } \\
(\mathrm{g})\end{array}$ & $\begin{array}{c}\mathrm{CV} \\
(\%)\end{array}$ \\
\hline Hembra & 9,043 & $151.4 \pm 39.7^{\mathrm{a}}$ & 26.20 \\
Macho & 8,131 & $154.7 \pm 42.6^{\mathrm{b}}$ & 27.62 \\
\hline \multicolumn{4}{c}{$\mathrm{CV}(\%)=$ coeficiente de variación } \\
a,b Diferentes superíndices indican & diferencia \\
significativa ( $(\mathrm{p}<0.05)$
\end{tabular}

$(p<0.05)$ y entre edad adulto sobre el sexo $(\mathrm{p}<0.05)$, pero no hay diferencia significativa entre edad juvenil sobre el sexo (Cuadro 9). Es decir, las vicuñas adultas machos producen más vellón sucio que las vicuñas hembras adultas, en tanto que las vicuñas juveniles no varían en su producción de PVS por efecto del sexo.

La producción de fibra de vicuña fue similar a los datos del estudio de Quispe et al. (2015) en la región Puno, Perú, donde en un periodo de dos décadas (1994-2014) se octuplicó la producción de fibra con un pro- medio anual de $715.7 \mathrm{~kg}$, con una tendencia ascendente sostenida y una tasa de crecimiento anual de $10.2 \%$. En cambio, Cabrera et al. (2010), trabajando con criadores de vicuña y guanaco en San Cristóbal de Ayacucho, Perú (1994-2009) obtuvieron $526.7 \mathrm{~kg}$ o un promedio de producción anual y una tasa de crecimiento anual de $5 \%$, pero con altibajos.

La literatura científica reporta diversos niveles de producción de fibra de vicuña. Así, Chambilla et al. (2015) reportan $159 \pm 28.3 \mathrm{~g}$ de PVS, Cabrera et al. (2010) de 197 g, Quispe et al., (2015) de 184 g, Maquera (2015) de 214.1 g, Cucho et al. (2012) de $182.6 \mathrm{~g}$, Quispe et al. (2010) de $189.7 \mathrm{~g}$, Choque (2010) de $230 \mathrm{~g}$ y Rigalt et al. (2006) de 416 g. Los resultados del presente estudio son menores a los reportados por los autores citados, probablemente debido al alto grado de dispersión de los PVS, los cuales oscilaron en el rango 54 a $426 \mathrm{~g}$. En este sentido, Maquera (2015) trabajó con un rango de 140 a 333 g, Quispe et al. (2010) de 96 a $356 \mathrm{~g} \mathrm{y}$ Cabrera et al. (2010) de 160 a 268 g. Por otro lado, esto estaría indicando que se estarían esquilando y obteniendo vellones con fi- 
Cuadro 9. Peso de vellón sucio (PVS) por edad y sexo entre los años 2008 y 2016 en la Región Apurímac, Perú

\begin{tabular}{ccccc}
\hline Edad & Sexo & Vicuñas $(\mathrm{n})$ & Promedio $(\mathrm{g})$ & CV $(\%)$ \\
\hline Adulto & Hembra & 6289 & $159.48 \pm 40.56^{\mathrm{a}}$ & 25 \\
& Macho & 4952 & $168.25 \pm 43.90^{\mathrm{b}}$ & 26 \\
\multirow{3}{*}{ Juvenil } & Hembra & 2754 & $132.94 \pm 31.09$ & 23 \\
& Macho & 3179 & $132.52 \pm 30.54$ & 23 \\
\hline
\end{tabular}

CV $(\%)$ = coeficiente de variación

${ }^{a, b}$ Diferentes superíndices entre edad indican diferencia significativa $(p<0.05$

bras muy cortas, y que la selección de vicuñas para esquilar no estaría siendo la adecuada. Quispe et al. (2009) recomienda realizar las campañas de Chacu cada dos años para obtener promedios de peso de vellón de hasta $250 \mathrm{~g}$ de fibra por vicuña. Por otro lado, Osnayo y Baquerizo (2015) indican que la longitud de fibra para vicuñas adultos macho y hembras fue de 16.5 y $15.6 \mathrm{~mm}$, respectivamente y para vicuñas juveniles machos y hembras fue de 19.9 y $19.8 \mathrm{~mm}$ en una comunidad de Apurímac, lo que refuerza las apreciaciones sobre la esquila y obtención de vellones con fibras muy cortas en la región de Apurímac.

Los resultados sobre la población de vicuñas de acuerdo con los sistemas de manejo están en relación con los reportados por Quispe et al. (2015) durante el Chacu 2014 en la región Puno, donde se capturaron 18217 vicuñas, de las cuales el $95 \%$ se capturaron en semicautiverio y el $5 \%$ en silvestría. Según Lichtenstein (2006), el sistema de manejo en semicautiverio mejora el aprovechamiento de la vicuña.

La población de vicuñas que pueden ser capturadas por grupo etario fueron similares al estudio de Quispe et al. (2015), quienes en su captura se encontró $75.4,10.9$ y $13.7 \%$, de vicuñas adultas, juveniles y crías, respectivamente. En lo que respecta a vicuñas esquiladas por edad, también se obtuvieron re- sultados acordes con Quispe et al. (2015) donde el $75 \%$ de animales esquilados fueron adultos.

Quispe et al. (2010) y Choque (2010) encontraron que las vicuñas de edad juvenil tienen menor PVS en comparación con las vicuñas de edad adulta. De la misma forma Quispe (2011) obtuvo un PVS para vicuñas adultas de $207.3 \pm 3.2 \mathrm{~g}$ y para vicuñas juveniles de $173.5 \pm 3.5 \mathrm{~g}$, probablemente debido a que los animales juveniles no tendrían el tiempo necesario para el desarrollo y crecimiento de su fibra. Además, Mueller et al. (2010) indica que el PVS aumenta en $15.6 \mathrm{~g} /$ año.

Los resultados de vicuñas capturadas y esquiladas según el sexo coinciden con Quispe et al. (2015), quienes reportaron capturas de 46 y $54 \%$ de vicuñas capturadas machos y hembras, respectivamente y de 49.5 y $51 \%$ vicunas esquiladas machos y hembras, respectivamente.

\section{Conclusiones}

La producción de fibra sucia de vicuña desde el año 2008 al 2016 presentó una tasa de crecimiento anual de $9.45 \%$, que representa una tasa de captura de $11.6 \%$. Además, se observó diferencias significativas para los efectos simples en la edad y sexo y sus interacciones. 


\section{Literatura Citada}

1. Bonacic C. 2000. Dinámica poblacional de la vicuña (Vicugna vicugna) y determinación de la capacidad de carga en la Provincia de Parinacota-Chile. En: Gonzalez B. Bas F, Tala C, Iriarte A. Manejo sustentable de la vicuña y el guanaco. Chile: Pontificia Universidad Católica de Chile. p 93-101.

2. Brack A. 1980. Conservación de la vicuña en el Perú. Información básica. Lima, Perú: Ministerio de Agricultura y Alimentación. 33 p.

3. Cabrera B, Infanzón A, Huamaní G 2010. Caso: asociación vicuña guanaco San Cristóbal y Aledaños (AVIGSCIA). En: Taller Nacional Experiencias de Gestión Participativa en Ecosistemas. Ayacucho, Lima, Perú.

4. Calle ER. 1982. Producción y mejoramiento de la alpaca. Lima, Perú: Fondo del Libro. Banco Agrario del Perú. 334 p.

5. Cajahuamán A. 2018. Análisis de la crianza de vicuñas en cautiverio en el parque conservacionista de Wislamachay: comunidad campesina San Antonio de Rancas - Pasco. Tesis de Ingeniero Zootecnista. Pasco: Univ. Nacional Daniel Alcides Carrión. 93 p.

6. Chambilla J, Víctor E, Gonzales V. 2015. Características productivas de la fibra de vicuña (Vicugna vicugna) en silvestría en el altiplano boliviano. La Paz, Bolivia: Unidad Académica Campesina de Tiahuanaco. UMSA.

7. Choque S. 2010. Caracterización física de la fibra de vicuña en los ayllus de la cuenca lago Poopo de Oruro Bolivia. En: III Simposio Internacional de Investigaciones sobre Camélidos Sudamericanos. Arequipa, Perú. DESCO.

8. Cucho H, Urquizo D, Ampuero E, Ordoñez, C, Alarcón V, Flores N. 2012. Características de fibra de vicuña del Centro Experimental La Raya Cusco. En: VI Congreso Mundial de Camélidos Sudamericanos. Arica, Chile.
9. Gimpel J, Bonacic C. 2006. Manejo sostenible de la vicuña bajo estándares de bienestar animal. En: Vilá B (ed). Investigación, conservación y manejo de vicuñas. Buenos Aires, Argentina. p. 113-132.

10. Lichtenstein G, OribeF, Grieg-Gran M, Mazzucchelli S. 2002. Manejo comunitario de vicuñas en Perú: estudio de caso de manejo comunitario de vida silvestre. PIE Series N ${ }^{\circ} 2$. UK: Earthprint Ltd. $82 \mathrm{p}$.

11. Lichtenstein G 2006. Manejo de vicuñas en cautiverio: el modelo del CEA INTA Abrapampa. En: Bila B (ed). Investigación, conservación y manejo de vicuñas. Argentina: Proyecto MACS. p 133-146.

12. Maquera FE. 2015. Características físicas de la fibra de vicuña en la zona alta andina de Tacna. Rev Investig Altoandin 17: 433-436. doi: 10.18271/ ria. 2015.157

13. [MINAGRI-DGFFS] Ministerio de Agricultura y Riego - Dirección General Forestal y de Fauna Silvestre. 2014. Censo poblacional de vicuñas 2012. [Internet]. Disponible en: http:// www.agrolalibertad.gob.pe/sites/default/ files/Censo $\% 20$ Poblaciona $1 \%$ 20de\%20Vicunas\%202012.pdf

14. [MINAGRI-DGFFS] Ministerio de Agricultura y Riego - Dirección General Forestal y de Fauna Silvestre. 2015. Lineamientos para el aprovechamiento y comercialización de la fibra de camélidos sudamericanos silvestres. [Internet]. Disponible en: https:// www.legislacionforestal.org/2017/09/25/ lineamientos-para-el-aprovechamientoy-comercializacion-de-la-fibra-decamelidos-sudamericanos-silvestres-rdeno-060-2015-serfor-de/

15. [MINAGRI] Ministerio de Agricultura y Riego, [SERFOR] Servicio Nacional Forestal y de Fauna Silvestre. 2015. Ley Forestal y de Fauna Silvestre 29763. Reglamento para la gestión de fauna silvestre. Lima, Perú. 90 p. 
16. Mueller JP, Rigalt F, Cancino AK, Lamas H. 2010. Calidad de las fibras de camélidos sudamericanos en Argentina. En: International Symposium on Fiber South American Camelids. Huancavelica, Perú.

17. Osnayo M, Baquerizo M. 2015. Determinación de la longitud y diámetro de la fibra de vicuña (Vicugna vicugna) por grupos etarios y sexo en la comunidad campesina de Lliupapuquio (Apurímac). Tesis de Médico Veterinario. Lima, Perú: Univ. Alas Peruanas. 94 p.

18. Quispe DM. 2011. Análisis de 1a interacción entre las poblaciones de los animales domésticos y de vicuñas ¿estabilización o competencia? en la Reserva Nacional Pampa Galeras. Tesis de Maestría. Lima, Perú: Univ. Nacional Agraria La Molina. $182 \mathrm{p}$.

19. Quispe EC, Rodríguez TC, Iñiguez LR, Mueller JP. 2009. Producción de fibra de alpaca, llama, vicuña y guanaco en Sudamérica. Anim Genetic Resources Information 45: 1-14. doi: 10.1017/ S1014233909990277

20. Quispe EC, Ramos H, Mayhua P, Alfonso L. 2010. Fibre characteristics of vicuña (Vicugna vicugna mensalis).
Small Ruminant Res 93: 64-66. doi:10.1016/j.smallrumres.2010.03.019.

21. Quispe EC, Butron BR Quispe, DM, Arratia MC. 2015. Producción de fibra de vicuña en semicautiverio y silvestria: tendencia, características y situación actual en la Región Puno. Rev Invest Altoandinas 17: 369-378.

22. Rabinovich JE, Capurro AF, Pessina LL. 1991. Vicuña use and the bioeconomics of an Andean peasant community in Catamarca, Argentina. In: Neotropical wildlife use and conservation. USA: University of Chicago Press. p 337-358.

23. Rigalt F, Rebuffi G, Vera R, Pivotto R. 2006. Caracterización preliminar de la calidad defibra de vicuña (Vicugna vicugna) de la Reserva Laguna Blanca. En: IV Congreso Mundial de Camélidos, Catamarca, Argentina.

24. Zúñiga M, Bujaico N. 2014. Empadre de vicuñas en el cerco permanente del Centro de Investigación, Producción y Transferencia Tecnológica, CIPTT Tullpacan-cha - Universidad Alas Peruanas, Huancavelica - Perú. Ciencia y Desarrollo 16: 21-24. doi: 10.21503/ CienciayDesarrollo.2013.v16i2.02 